\title{
Measurement of low frequency mechanical vibrations based on an inverted magnetic pendulum
}

\author{
Susana V. Awad, Joaquin F. Orozco, Fredy E. Hoyos \\ Facultad de Ciencias-Escuela de Física, Universidad Nacional de Colombia-Sede Medellín, Colombia
}

\begin{tabular}{|c|c|}
\hline Article Info & ABSTRACT \\
\hline Article history: & In this paper is presented the mathematical model, design and construction of \\
\hline Received Jan 19, 2019 & $\begin{array}{l}\text { a prototype of a vibration frequency meter in an adjustable range of } 2 \mathrm{~Hz} \text { to } \\
30 \mathrm{~Hz} \text {; The experimental results and their analysis are also presented, making }\end{array}$ \\
\hline Revised Apr 9, 2019 & a comparative evaluation with the theoretical model. The device is based on \\
\hline Accepted Apr 21, 2019 & $\begin{array}{l}\text { the principle of resonance applied in an inverted magnetic pendulum whose } \\
\text { natural frequency can be modified by variations of physical parameters. }\end{array}$ \\
\hline Keywords: & $\begin{array}{l}\text { The oscillation of the pendulum is recorded detecting variations in the } \\
\text { magnetic field using hall effect sensors; the data recorded with a }\end{array}$ \\
\hline Frequency meter & microprocessor is analyzed and the results are simultaneously plotted in a \\
\hline Hall effect & $\begin{array}{l}\text { computer interface. The data obtained were processed to be plotted in the } \\
\text { frequency domain. facilitating its analysis. It was proved that the prototype }\end{array}$ \\
\hline Magnetic pendulum & can be used as a frequency meter and that the adjustable character of the \\
\hline Seismometer & device works according to the mathematical model. Finally, The effect of the \\
\hline Wide working bandwidth & $\begin{array}{l}\text { friction force was studied, it was concluded that the friction force affects the } \\
\text { measurement after a considerable period of time of oscillation, but not in the } \\
\text { first moments. }\end{array}$ \\
\hline
\end{tabular}

Copyright $(0) 2019$ Institute of Advanced Engineering and Science. All rights reserved.

\section{Corresponding Author:}

Fredy E. Hoyos,

Facultad de Ciencias - Escuela de Física,

Universidad Nacional de Colombia - Sede Medellín,

Carrera 65 No. 59A-110, Medellín, Colombia.

Email: fehoyosve@unal.edu.co

\section{INTRODUCTION}

Seismic detection is a subject that has been prevalent in research during the last decades. The telluric movements generate elastic waves on the surface of the earth with frequencies below the millihertz and up to $30 \mathrm{~Hz}$, higher frequencies are attenuated promptly after traveling short distances [1]. The seismometers are the key sensing elements to detect seismic signals in the fields of geophysical prospection and seismic monitoring [2]. A variety of seismometers were developed, such as the electromagnetic moving-coil seismometers [3], capacitive seismometers [4], optical fiber seismometers [5], micro- electromechanical systems (MEMS) seismometers [6], and electrochemical seismometers [7, 8]. The processing of seismic signals has been undoubtedly the motivation and driving factor of many innovations in the signal processing area, remarkably in blind deconvolution, time frequency distributions, and even neural networks [9-14]. The operation of a classic seismic sensor consists, precisely, in the obtaining of signals from the displacement of a suspended mass due to the inertial force of the movement of the ground. This principle is used in low frequency seismometers, where the output signal is proportional to the displacement and the speed of the mass. Contrarily, broadband seismometers use a feedback force which compensates the inertial force and by means of a suitable control system the acceleration of the soil can be calculated [15].

The accurate detection of vibration signal plays an important role in many fields such as on-line monitoring, machinery, vehicles, earthquake monitoring, navigation system in aerospace equipment, the calibration of instruments such as shaking tables [16-18] and the review of the status of different civil 
structures [9], especially in those structures in which the natural frequencies can coincide with the frequency of movement of people, representing a typical case of resonance [19, 20].

By the whole of low frequency seismometers, the design of horizontal seismic sensors based on an inverted magnetic pendulum [21, 22] has been described, where an optical mouse senses the pendulum movement, but with that kind of sensor a correct reading of the data was not achieved. In addition, the design of that seismometer can only be used in a particularly small frequency range (close to its natural frequency); which means it's not a versatile frequency meter [18]. Another way to monitor the vibrations of civil structures is electronically using optical fibers, this methodology has had satisfactory results thanks to the precision that it involves, the main problem with this methodology is that the cost is high, which results in it not being affordable for the population of developing countries.

This paper presents the design and evaluation of a device in order to measure mechanical vibrations based on an inverted magnetic pendulum [23] that has resonance under the action of a frequency lower than $30 \mathrm{~Hz}$; improving some characteristics of similar prototypes such as the resolution of the measurements [24], the accuracy of the signal captured and the cost of the design. Four Hall effect sensors will be used to sense the frequency of mechanical vibration.

The paper is organized as follows: section 2 presents the mathematical model and schematic of the inverted magnetic pendulum in section 3 the materials and methods are presented, section 4 shown the experimental results and section 5 presents conclusions of the work.

\section{MATHEMATICAL MODEL}

Rohmanuddin (3) formulates the design of a low frequency seismic sensor based on the mathematical properties of the pendulum:

A pendulum consists of a mass $M$ suspended and supported by a stem of length $L$ and a negligible mass anchored at one of its ends. It could be modeled, for small angles, by the next differential equation:

$$
\frac{d^{2} \theta}{d t^{2}}+\frac{g}{L} \theta=0
$$

which corresponds to a harmonic oscillator with frequency:

$$
f=\frac{1}{2 \pi} \sqrt{\frac{g}{L}}
$$

In the hypothetical case where the gravity acts in the opposite direction on the pendulum (inverted pendulum), the sign of the quotient, which is within the root, would change, as described in (3):

$$
f=\frac{1}{2 \pi} \sqrt{-\frac{g}{L}}=\frac{j}{2 \pi} \sqrt{\frac{g}{L}}
$$

This sign's change is an indication to mesure disturbances caused due to forces with low frequencies. For the inverted pendulum is required a a restoring force which forces the mass to remain suspended and, consequently, let the mass oscillate around a point of equilibrium.

The force on a magnetic dipole $\vec{m}$, whic is placed in a magnetic field $\vec{B}$ :

$$
\vec{F}=\nabla(\vec{m} \cdot \vec{B})
$$

To find the magnetic field generated by one of the magnets, it was considered the potential due to a magnetic dipole.

$$
\vec{A}(\vec{r})=\frac{\mu_{0}}{4 \pi} \frac{\vec{m} \times \vec{r}}{r^{2}}
$$

For a magnetic dipole which is situated at the origin and points toward the direction of $\hat{z}$ the potential in the point $(r, \theta, \phi)$ is given by,

$$
\vec{A}(\vec{r})=\frac{\mu_{0}}{4 \pi} \frac{m \sin (\theta)}{r^{2}} \hat{\phi}
$$

From the above expression, the magnetic field can be written as:

$$
\vec{B}=\nabla \times \vec{A}=\frac{\mu_{0} m}{4 \pi r^{3}}(2 \cos (\theta) \hat{r}+\sin (\theta) \hat{\theta})
$$


In the case where the displacements around the $z$ axis are small enough, its considered $\theta=0$ :

$$
\vec{B}=\frac{\mu_{0} m}{2 \pi z^{3}} \hat{z}
$$

Then, for two identical magnetic dipoles situate along the $\mathrm{z}$ axis, which have their opposite poles faced.

$$
\begin{aligned}
& \vec{m} \cdot \vec{B}=\frac{\mu_{0} m^{2}}{2 \pi z^{3}} \\
& \vec{F}=\frac{\partial}{\partial z}\left[\frac{\mu_{0} m^{2}}{2 \pi z^{3}}\right] \hat{z}=-\frac{3 \mu_{0} m^{2}}{2 \pi z^{4}} \hat{z}
\end{aligned}
$$

A diagram of the inverted pendulum is shown in the Figure 1.

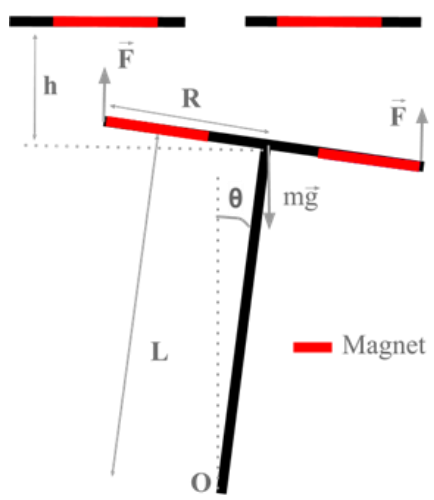

Figure 1. Schematic of the inverted magnetic pendulum

Analyzing the torque around de point $O$, it is deduced the equation of movement which is given by

$$
L^{2} m \frac{d^{2} \theta}{d t^{2}}+\left(-m g L+\frac{8 k R^{2}+2 k h L}{h^{3}}\right) \theta=0
$$

where $k$ is an auxiliary constant used to simplify the expression obtained from the equation (10),

$$
k=\frac{3 \mu_{0} m^{2}}{2 \pi} .
$$

Therefore, the equation of movement corresponds to a harmonic oscillator with frecuency:

$$
f=\frac{1}{2 \pi} \sqrt{\frac{8 k R^{2}+2 k h L}{h^{3} L^{2} m}-\frac{g}{L}}
$$

To achieve low frequencies in this inverted pendulum model it is necessary to calculate the minimum real number for the espression within the root in the equation (12). It is also necessary to vary ,properly, the parameters in order to obtain different natural frequencies of the system.

\section{MATERIALS AND METHODS}

\subsection{Design of the device}

Based on the mathematical properties of the inverted magnetic pendulum, it is constructed a device to detect and study mechanical vibrations, attaching the base of the pendulum to a source of vibration and due to the inertia of the levitating mass, the suspended magnet will leave the equlibrium position, that movement establishes a change in the magnetic field flux in space, which can be detected by a hall effect sensor, what corresponds to an electrical signal according to the (13).

$$
V_{H}=\frac{I B}{n t e}
$$


where nte are constants of the sensor, the signal $V_{H}$ is proportional to the magnitude of the magnetic field which crosses it $B$ and it is also proportional to the current through the sensor $I$. Assuming that, in the moment when the upper magnet leaves the equilibrium position, the variations of the magnetic field $B$ are small and linear regarding the space; therefore, it could be stablish a linear relation between the displacement of the magnet and the electrical signal $V_{H}$, that relation is also related with the source of the vibration that which had forced the magnet to leave the equilibrium point.

Furthermore, with the proposed device it is possible to set a certain natural frecuency of oscillation according to the configuration of the parameters of the equation (9). The design of the divice allows to adjust at least two parameters: the length of the stem $L$ and the distance between the magnets $h$. The length of the stem is adjustable by means of a system consisting of two axially aligned aluminum tubes, where one of the tubes is inserted into the other and can be fixed when it is in the desired length. The distance between the magnets can be adjusted due to a system of vertical networks in the structure of the device, which allows establishing different distances between the platforms that hold the magnets and sensors.

\subsection{Data acquisition system}

The signal from four Hall sensors (A1301) is digitized using an Arduino UNO card, which is based on the ATMega328 microcontroller, and then the information is sent to a computer through serial communication, this data is subsequently processed by a software programmed in PYTHON®; it should be clarified that the reading of the information is not done in parallel but sequentially, however the effect of delay between signals is negligible for this purpose. The general structure of the system can be appreciated in the diagram represented in the Figure 2.

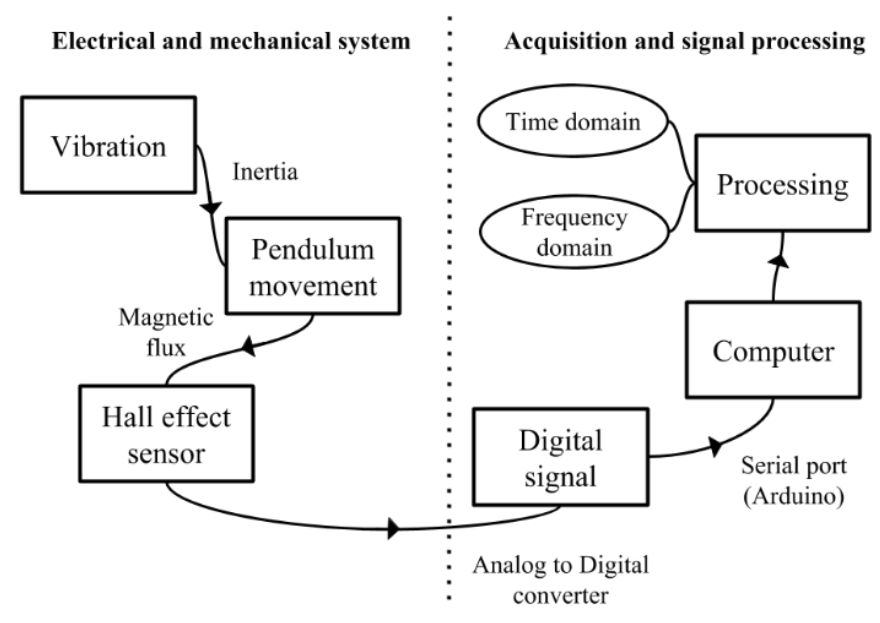

Figure 2. Structure of the system

Using the same software in PYTHON®, the acquired information is stored and real-time graphs of the oscillation of the pendulum are generated.Thereafter, the stored information of a given sample, for example with $\mathrm{N}$ data, can be analyzed using the discrete Fourier transform (DFT) defined as:

$$
X(f)=\sum_{k=0}^{N-1} x_{k} e^{-j 2 \pi f / N}
$$

What offers information about the vibration spectrum of a certain source, this process is done through the algorithm of the Fast Fourier Transform (FFT) that is included in the library SCIPY. Besides, since the pendulum has a certain natural frequency, due to the resonance phenomenon, those frequencies close to the natural frequency of the pendulum will be favored, which allows to contradistinguish possible sources of noise outside of the range of interest.

\subsection{Prototype}

The built prototype has ring-shaped ceramic magnets in the inverted pendulum, these magnets are supported on flat acrylic platforms whose relative distance can be adjusted between 0.5 and $4 \mathrm{~cm}$, approximately. The length of the stem, which is composed of two aluminum tubes, is adjustable between 10 and $20 \mathrm{~cm}$. The device has four Hall effect sensors located crosswise on a plane parallel to the platform that

Measurement of low frequency mechanical vibrations based on an inverted magnetic ... (Susana V. Awad) 
holds the upper magnet. An Arduino UNO card is attached to the upper magnet together with a functioning indicator system composed of LED's. The casing is made of acrylic that is a suitable material and easy to manufacture, the dimensions of the device are $10 \mathrm{~cm} \times 10 \mathrm{~cm} \times 30 \mathrm{~cm}$ as shown in the Figure 3 .

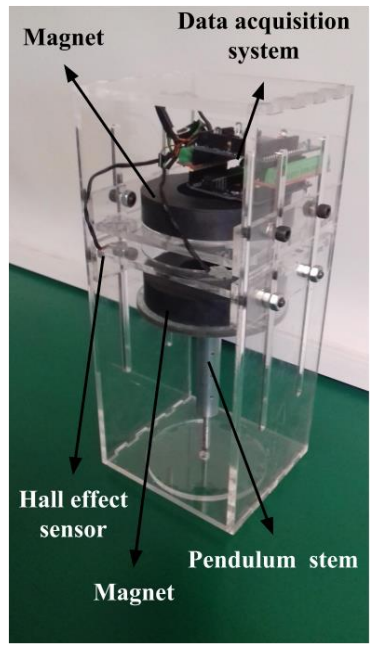

Figure 3. Prototype

\section{EXPERIMENTAL RESULTS}

In order to validate the running of the device, certain experiments were performed in which the base of the pendulum was excited by a speaker as a generator of mechanical oscillations at a certain frequency. The natural frequency of inverted pendulum is established for tests equal to $1.8 \mathrm{~Hz}$. In the Figure 4 it is shown the behavior of the pendulum oscillating after pushing the lower magnet out of equilibrium (at the start). Sinusoidal oscillations are obtained which, after a while, are slightly damped, decreasing the amplitude; this damping condition could be associated with friction at the base of the pendulum.

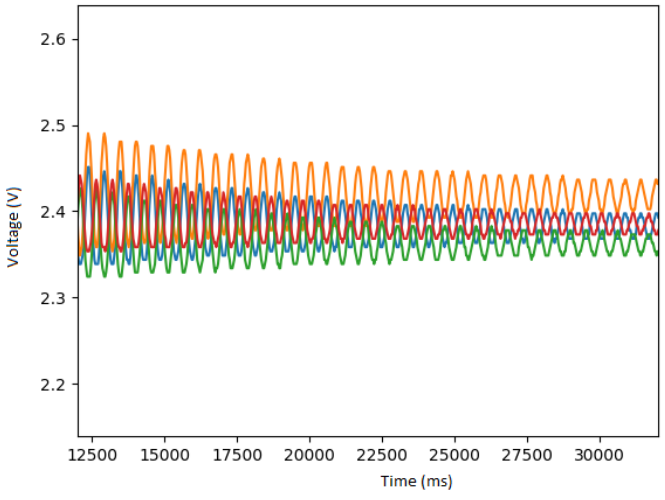

Figure 4. Real time plot- natural frecuency

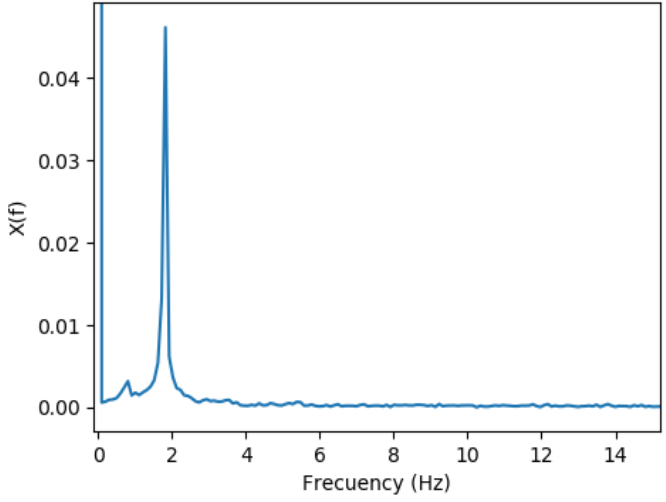

Figure 5. Vibration spectrum- natural frecuency

Subsequently, a discrete Fourier transform is applied to one of the signals, which is presented in the Figure 5. Aditionally the behavior of the system is evaluated when it is subjected to a vibration of $1 \mathrm{~Hz}$, the Figure 6 and Figure 7 show that the pendulum oscillates in a sinusoidal manner, while the amplitude increases from equilibrium to stabilize at a certain value. The spectrum shows some peaks just in the frequency of the exciter signal and predominantly in the natural frequency of the device. 


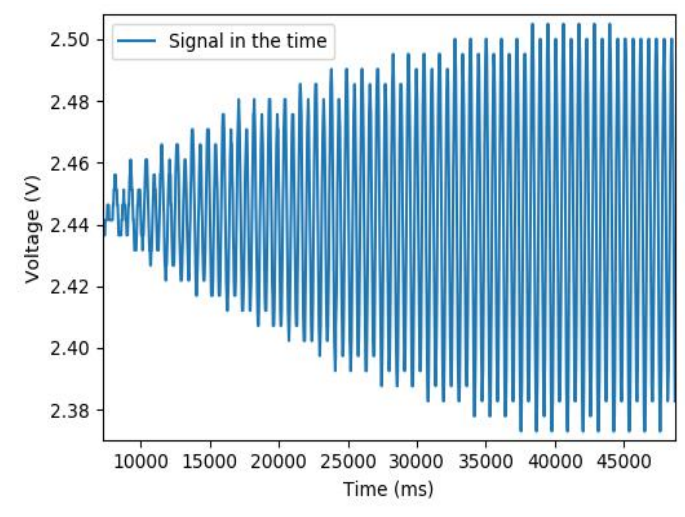

Figure 6. Real time plot- $1 \mathrm{~Hz}$

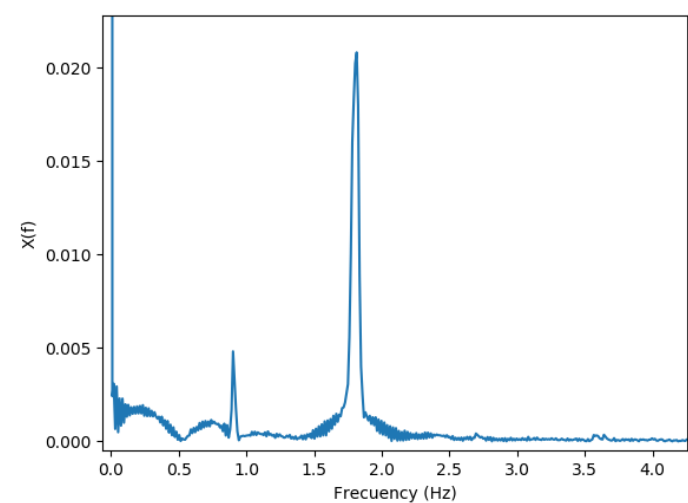

Figure 7. Effects of selecting different switching under dynamic condition

A similar effect is observed when the pendulum is excited with a vibration of $2 \mathrm{~Hz}$, the Figure 8 and Figure 9 show that the stabilization of the oscillation is achieved after a short period of time and the frequency of the source stands out in the spectrum, the stabilization in the amplitude of oscillation indicates that there is an influence of a retarding force, which is associated with the friction.

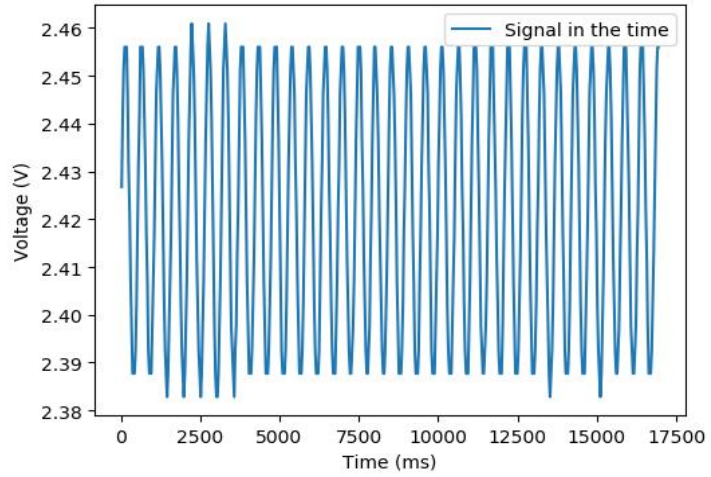

Figure 8. Real time plot- $2 \mathrm{~Hz}$

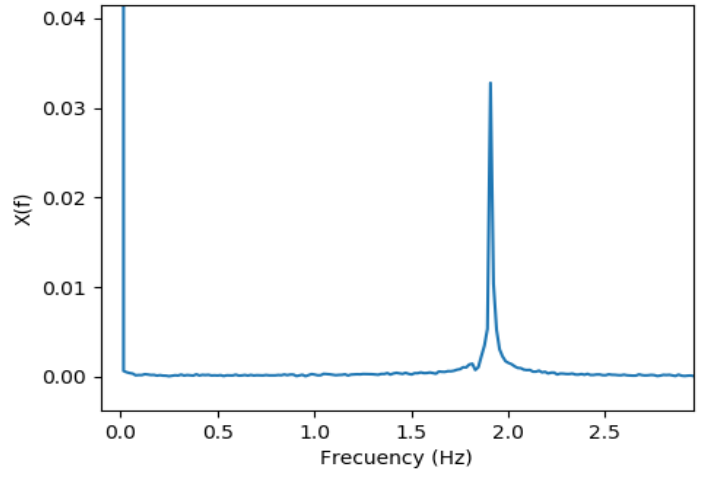

Figure 9. Vibration spectrum- $2 \mathrm{~Hz}$

Finally, when the pendulum is subjected to a vibration certainly different from the natural frequency, in this case $50 \mathrm{~Hz}$, the system does not experience any appreciable movement, this is evidenced in the Figure 10, where it is observed that the pendulum remains in the equilibrium position with remarkably slight vibrations compared with those observed in the previous cases.

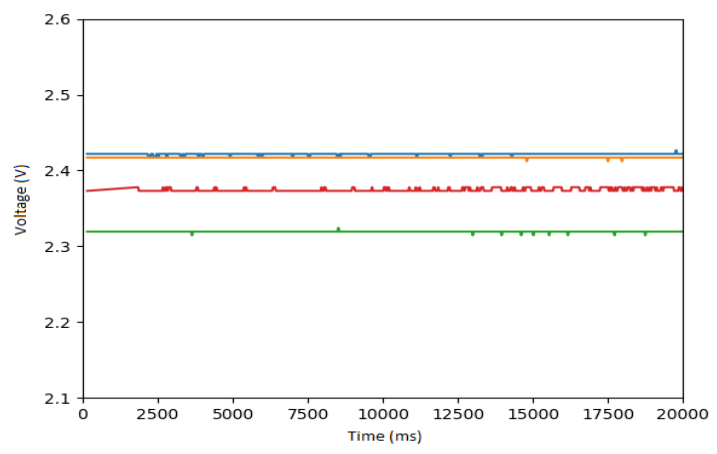

Figure 10. Real time plot- $50 \mathrm{~Hz}$ 


\section{CONCLUSION}

The experimental seismometer allows analyzing the oscillation spectrum of mechanical sources, especially those that are weak or distant in the frequency range between 1 and $30 \mathrm{~Hz}$. The system can be scaled as a low-cost system for technological applications, such as seismic monitoring, vibration analysis in structures and calibration of oscillating instruments. The friction, acting as a retarding force, affects the system in the long term, however, the measurement of the frequency can be performed accurately. By having a comparison of the results of the calibration of the prototype with the theoretical values of the mathematical model, its functioning as a versatile and adjustable device was satisfactorily verified.

\section{ACKNOWLEDGEMENTS}

The authors thank to the School of Physics, for the valuable support to conduct this research. This work was supported by the Universidad Nacional de Colombia, Sede Medellín under the projects HERMES-34671 and HERMES-36911.

\section{REFERENCES}

[1] S. Ingate and J. Berge, "Prospects for Low Frequency Seismometry," A report of the iris broadband seismometer workshops, pp. 24-26, Mar 2004.

[2] Z. Sun, et al., "A High-Consistency Broadband MEMS-Based Electrochemical Seismometer With Integrated Planar Microelectrodes," in IEEE Transactions on Electron Devices, vol/issue: 64(9), pp. 3829-3835, Sep 2017.

[3] G. H. Zhang and S. Y. Hu, "Dynamic characteristics of moving-coil geophone with large damping," Int. J. Appl. Electromagn. Mech., vol/issue: 33(1), pp. 565-571, Jan 2010.

[4] J. Laine and D. Mougenot, "Benefits of MEMS based seismic accelerometers for oil exploration," Proc. Transducers Conf., pp. 1473-1477, Jun 2007.

[5] T. C. Liang and Y. L. Lin, "Fiber optic sensor for detection of ground vibrations," Proc. SPIE, vol/issue: 8351(9), pp. 835109, Jan 2012.

[6] D. Mougenot and N. Thorburn, "MEMS-based 3D accelerometers for land seismic acquisition: is it time," Lead. Edge, vol/issue: 23(3), pp. 246-250, Mar 2004.

[7] V. G. Krishtop, et al., "Technological principles of motion parameter transducers based on mass and charge transport in electrochemical microsystems," Russian J. Electrochem., vol/issue: 48(7), pp. 746-755, 2012.

[8] W. T. He, et al., "MEMS based broadband electrochemical seismometer," Opt. Precis. Eng., vol/issue: 23(2), pp. 444-451, Feb 2015.

[9] F. Peng, et al., "Sensitivity Prediction of Multiturn Fiber Coil-Based Fiber-Optic Flexural Disk Seismometer via Finite Element Method Analysis,” Technology, vol/issue: 35(18), pp. 3870-3876, Sep 2017.

[10] I. Rodriguez, et al., "Signal processing in ocean bottom seismographs for refraction seismology," in IEEE Transactions on Instrumentation and Measurement, vol/issue: 55(2), pp. 652-658, Apr 2006.

[11] M. Gaeta, et al., "Blind separation of sources applied to convolutive mixtures in shallow water," Proc. IEEE Signal Processing Workshop Higher-Order Statistics, pp. 340-343, 1997.

[12] X. Zhang, et al., "Signal study on blind separation of underwater acoustic signals," Proc. Int. Conf. Signal Processing (WCCC-ICSP), vol. 3, pp. 1802-1805, 2000.

[13] Boles P. J. and Boashash B., "Applications of the cross-Wigner-Ville distribution to seismic data processing," in Boashash B., editor, "Time-Frequency Signal Analysis: Methods and Applications," Wiley, New York, NY, USA, pp. 445-456, 1992.

[14] Setayeshi, et al., "Underwater signal prediction and parameter estimation using artificial neural networks," Proc. Electrical and Computer Engineering, vol. 2, pp. 621-626, 1995.

[15] F. Acernese, et al., "A new architecture for the implementation of force-feedback tunable mechanical monolithic horizontal sensor," 2010 17th IEEE-NPSS Real Time Conference, 2010.

[16] M. Bernal, et al., "Control systems for shake tables: A critical review," Universidad Militar Nueva Granada (Colombia). Ingeniería y desarrollo, vol/issue: 33(2), 2015.

[17] T. Trombetti, "Experimental/analytical approaches to modeling, calibrating and optimizing shaking table dynamics for structural dynamic applications," Ph.D. Thesis, Department of Civil Engineering, Rice, 1998.

[18] M. Rohmanuddin, et al., "Design of horizontal seismic sensor with spherical inverted pendulum and magnetic levitation," 2011 2nd International Conference on Instrumentation Control and Automation, 2011.

[19] A. R. Ortiz, et al., "Efecto de la Interacción Humano-Estructura en las propiedades dinámicas de una tribuna," Revista Ingeniería y Competitividad, vol/issue: 14(1), pp. 63-73, 2012.

[20] S. Zivanovic, et al., "Vibration serviceability of footbridges a under Human-Induced excitation: a literarure review," Journal of Sound and Vibrations, pp. 1-74, 2005.

[21] P. T. Tin, et al., "Using real interpolation method for adaptive identification of nonlinear inverted pendulum system," Int. J. Electr. Comput. Eng., vol/issue: 9(2), pp. 1078-1089, Apr 2019.

[22] M. A. A. Al-Mekhlafi, et al., "Adaptive Neuro-Fuzzy Control Approach for a Single Inverted Pendulum System," Int. J. Electr. Comput. Eng., vol/issue: 8(5), pp. 3657, Oct 2018. 
[23] E. H. Karam, et al., "Design Nonlinear Model Reference with Fuzzy Controller for Nonlinear SISO Second Order Systems," Int. J. Electr. Comput. Eng., vol/issue: 9(4), Aug 2019.

[24] F. E. Hoyos, et al., "Selection and validation of mathematical models of power converters using rapid modeling and control prototyping methods," Int. J. Electr. Comput. Eng., vol/issue: 8(3), pp. 1551, Jun 2018.

\section{BIOGRAPHIES OF AUTHORS}

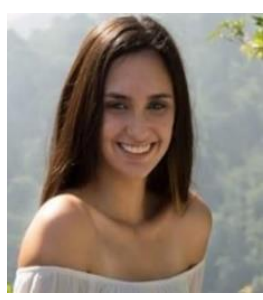

Susana V. Awad: Physical engineering student, Science Faculty, School of Physics, Universidad Nacional de Colombia, Sede Medellín, Colombia. She has held positions of academic support to other students in basic science subjects. Her research interests are mainly simulations of chemical physical processes and the reading and processing of signals.

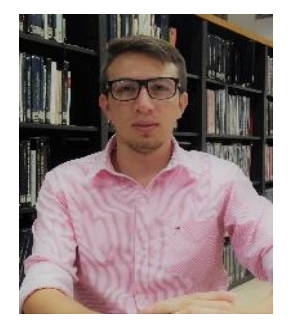

Joaquin F. Orozco: Undergraduate student in physical engineering, Science Faculty, Universidad Nacional de Colombia, Medellín, Colombia. Member of the reasearch group in Digital Signal Processing for Real-Time Systems at the same university. His research interests include applied electronics and physics.

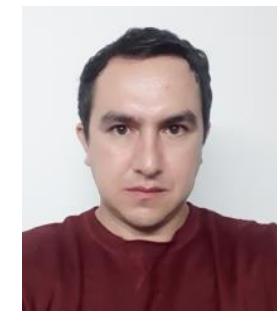

Fredy Edimer Hoyos: received his BS and MS degree from the National University of Colombia, at Manizales, Colombia, in Electrical Engineering and Industrial Automation, in 2006 and 2009, respectively, and Industrial Automation Ph.D. in 2012. Dr. Hoyos is currently an Associate Professor of the Science Faculty, School of Physics, at National University of Colombia, at Medellin, Colombia. His research interests include nonlinear control, system modelling, nonlinear dynamics analysis, control of nonsmooth systems, and power electronics, with application within a broad area of technological process. Dr. Hoyos is an Associate Researcher in Colciencias and member of the Applied Technologies Research Group - GITA at the Universidad Nacional de Colombia. https://orcid.org/0000-0001-8766-5192 